\title{
Anti-CD47 Monoclonal Antibody SRF231
}

National Cancer Institute

\section{Source}

National Cancer Institute. Anti-CD47 Monoclonal Antibody SRF231. NCI Thesaurus. Code C155976.

A human monoclonal antibody targ eting the human cell surface antigen CD47, with potential phagocytosis-inducing and antineoplastic activities. Upon administration, antiCD47 monoclonal antibody SRF231 selectively binds to CD47 on tumor cells and blocks the interaction of CD47 with signal regulatory protein alpha (SIRPalpha), an inhibitory protein expressed on macrophages. This prevents CD47/SIRPalpha-mediated signaling and abrog ates the CD47/SIRPa-mediated inhibition of phagocytosis. This induces prophagocytic signaling mediated by the binding of calreticulin (CRT), which is specifically expressed on the surface of tumor cells, to low-density lipoprotein $(L D L)$ receptor-related protein (LRP), expressed on macrophages. This results in macrophage activation and the specific phagocytosis of tumor cells. In addition, blocking CD47 signaling activates both an anti-tumor T-lymphocyte immune response and T cell-mediated killing of CD47expressing tumor cells. CD47, also called integrin-associated protein (IAP), is a tumorassociated antigen (TAA) expressed on normal, healthy hematopoietic stem cells (HSCs) and overexpressed on the surface of a variety of cancer cells. Expression of CD47, and its interaction with SIRPalpha, leads to the inhibition of macrophage activation and protects cancer cells from phagocytosis, which allows cancer cells to proliferate. 